\title{
Shape evolution and bubble formation of acoustically levitated drops
}

Wenli Di, ${ }^{1 \#}$ Zehui Zhang, ${ }^{2 \#}$ Lin Li, ${ }^{2 \#}$ Kejun Lin, ${ }^{1}$ Jun Li ${ }^{1}$, Xiaoguang Li, ${ }^{1}$ Bernard P. Binks, ${ }^{3}$ Xiaopeng Chen ${ }^{2}$ and Duyang Zang ${ }^{1 *}$

1 Functional Soft Matter \& Materials Group, Key Laboratory of Space Applied Physics and Chemistry of the Ministry of Education, School of Science, Northwestern Polytechnical University, Xi'an, 710129, China

2 School of Mechanics, Civil Engineering and Architecture, Northwestern Polytechnical University, Xi'an 710129, China

3 School of Mathematics and Physical Sciences, University of Hull, Hull. HU6 7RX. UK

*Corresponding author: Duyang Zang Tel:+86-29-88431618 dyzang@nwpu.edu.cn

\# The authors contributed equally to the work

Revised version sent to Physical Review Fluids on 10.9.18

The published version of this article can be accessed at https://doi.org/10.1103/PhysRevFluids.3.103606 


\begin{abstract}
In this study, we investigated the shape evolution and bubble formation of acoustically levitated drops upon increasing the sound intensity. Here, a levitated liquid drop evolves progressively from an oblate spheroidal shape to a flattened film to a thin bowl-shaped film, eventually forming a closed bubble. Through systematic experiments, numerical simulation and scaling analysis, we demonstrate that the buckled geometry of the liquid film can drastically enhance the suction effect of acoustic radiation pressure at its rim, forming a significant pressure gradient inside the film which causes an abrupt area expansion and bubble formation. Our results provide the mechanical origin responsible for the shape evolution and bubble formation of acoustically levitated drops, and highlight the role of buckled geometry in the levitation and manipulation of liquid films in an ultrasound field.
\end{abstract}

Keywords: Acoustic levitation, droplet, bubble, surface tension 


\section{Introduction}

Owing to the non-linear effect of ultrasound, foreign objects ranging from solid particles to liquid drops can experience a steady time-averaged force when irradiated by an ultrasound field, which is referred to as acoustic force[1]. By utilizing the acoustic radiation force of a standing sound wave field, acoustic levitation provides one of the most powerful techniques for the study of drop dynamics and physics[2-5], as well as drop manipulation[6, 7]. The static shape of the acoustically levitated drop is determined by the balance between the acoustic radiation pressure $P_{\mathrm{A}}$, surface tension $\sigma$ and its internal pressure $P_{\mathrm{i}}$, and can be written as[8]

$$
P_{\mathrm{i}}-P_{\mathrm{A}}=\sigma \nabla \cdot \mathbf{n}
$$

where $\underline{P}_{\mathrm{A}}$ is a time-averaged pressure caused by ultrasound, $\mathbf{n}$ is the normal unit vector on the drop surface pointing outward and $\nabla \cdot \mathbf{n}$ is the total local curvature. However, the levitated drop often exhibits various kinds of dynamic behavior. The competition between the internal pressure of the drop and the Bernoulli pressure caused by sound inevitably leads to radial oscillation[8]. In the single-axis levitator, solid objects often exhibit spontaneous oscillation in both the radial and axial directions[9], which was well explained by a simple model based on a harmonic oscillator. Similarly, acoustically levitated liquid drops can undergo a vertical vibration if it is deviated from its equilibrium levitation position, which also shows a harmonic mode[10]. The vertical oscillation can probably be damped via the acoustic streaming in the sound field[11]. Moreover, the levitated drops can exhibit many types of instability including 
atomization[12], breakup[13] and buckling[8, 14].

Buckling instability of the acoustically levitated drop was visualised by the buckling of the flattened film followed by rapid area expansion, first reported by Lee et al.[8]. This phenomenon could be triggered by increasing the sound intensity or by heating the levitated drop using a laser as reported by Pathak and Basu[14]. Recently, Zang et al. have found that similar bubble formation can be triggered by buckling the liquid film in a controlled manner, and they attributed this phenomenon to the acoustic resonance of the opening cavity encapsulated by the buckled film with the sound field in the levitator[15]. The acoustic resonance mechanism explained well why the abrupt area expansion occurred at a critical cavity volume and was independent of the type of liquid. However, the force exerted on the acoustically levitated drop that drives its shape evolution was not understood clearly. A force analysis based on the calculation of sound field and acoustic radiation pressure on the drop surface is highly desirable. In the present work, we study the shape evolution of acoustically levitated drops upon increasing the sound intensity, particularly investigating the buckling of the flattened liquid film and the bubble formation phenomenon triggered by the sound field. We focus on the spatial distribution of acoustic radiation pressure exerted on the liquid surface and how it impacts on drop shape evolution.

\section{Materials and methods}

\section{A. Experimental setup and method}

The single-axis acoustic levitator (SonoRh-1, Shengdu Ltd., China) consists of an 
emitter and a reflector which were arranged coaxially with respect to the gravitational direction. The frequency of the emitter was $20.7 \mathrm{kHz}$. The cross-sectional radii of the emitter and reflector, $r_{1}$ and $r_{2}$, were 12.5 and $25.0 \mathrm{~mm}$, respectively. The surface of the reflector was concave, with a radius of curvature $r_{3}=59.4 \mathrm{~mm}$. The details can be found elsewhere[16].

In order to conveniently adjust the sound intensity in the levitator, the reflector was fixed on a micro-lifting table (ST401ES60, Strong Precision, China) to regulate the distance $D$ between the emitter and reflector. The lift rate of the reflector $u_{\mathrm{R}}$ can be accurately controlled by a servo motor (42BYGH47-1684B, Sihongmotor, China). In our experiments, $u_{\mathrm{R}}$ was fixed at $1.0 \mathrm{~mm} / \mathrm{s}$. The distance $D$ was varied from 45.0 to 40.0 mm. The dynamics of the levitated droplets was recorded by a high-speed CCD camera (Photron Fastcam Mini UX100, Japan) at 2,000 frames per second. The experiments were performed at $\sim 20^{\circ} \mathrm{C}$ and relative humidity of $\sim 40 \%$.

\section{B. Materials}

Different liquids including water, aqueous solutions of sodium dodecyl sulfate (SDS), poly(ethylene oxide) (PEO, molecular weight $\sim 10^{6} \mathrm{amu}$ ), glycerin and sucrose, a liquid crystal (4-cyano-4'-pentylbiphenyl, 5CB), PDMS silicone oil (20 mPa s) as well as a liquid Ga-In-Sn alloy were used for acoustic levitation. The water used was purified with an Ultrapure Water System (EPED, China). All other liquid samples were purchased from Aladdin Industrial Corporation, China. The surface tension of the liquid was measured with a Wilhelmy plate using a Langmuir trough instrument (JML04C3, 
Powereach Ltd., China). The viscosity of the liquid was measured with a stresscontrolled rheometer (Physica MCR 302, Anton Paar, Germany) equipped with a coneand-plate geometry. Some properties of the liquids are summarized in Table 1.

Table 1. Properties of the different liquids at $22{ }^{\circ} \mathrm{C}$ used in the experiments.

\begin{tabular}{lllll}
\hline & $\begin{array}{l}\text { Concentration/ } \\
\mathrm{g} / \mathrm{L}\end{array}$ & $\begin{array}{l}\text { Density/ } \\
\mathrm{g} \mathrm{cm}^{-3}\end{array}$ & $\begin{array}{l}\text { Viscosity, } \\
\eta / \mathrm{mPa} \mathrm{s}\end{array}$ & $\begin{array}{l}\sigma / \\
\mathrm{mN} \mathrm{m}^{-1}\end{array}$ \\
\hline Water & - & 0.998 & 0.90 & 72.60 \\
SDS-1 solution & $1.84(\sim 0.8 \mathrm{cmc} *)$ & 0.994 & 1.14 & 31.20 \\
SDS-2 solution & $2.30(\sim 1 \mathrm{cmc})$ & 0.994 & 1.15 & $\underline{32.55}$ \\
SDS-3 solution & $23(\sim 10 \mathrm{cmc})$ & 0.994 & 1.34 & 34.80 \\
5CB & - & 1.008 & 40.3 & 35.95 \\
PEO solution & 0.50 & 1.008 & 1.30 & 61.93 \\
Glycerin solution & $73 \mathrm{wt} . \%$ & 1.189 & 26.31 & 65.55 \\
Sucrose solution & $40 \mathrm{wt} \%$ & 1.128 & 2.49 & 81.86 \\
Silicone oil & - & 0.963 & 20 & 21.00 \\
Ga-In-Sn & - & 6.44 & 240 & $718^{[17]}$ \\
\hline
\end{tabular}

$* \mathrm{cmc}=$ critical micelle concentration

\section{Calculation of sound pressure field}

The sound pressure field was numerically calculated using a commercial finite element software Comsol Multiphystics 5.3. The simulation domain was determined by the geometry of the acoustic levitator. The boundary condition between the simulation domain and ambient was established as plane wave radiation. The boundary acoustic pressure was restrained by the Helmholtz equation:

$$
\nabla \cdot\left(-\frac{1}{\rho_{0}} \nabla p\right)-\frac{\omega^{2} p}{\rho_{0} c_{0}^{2}}=0
$$


where $p$ is the sound pressure, $\rho_{0}$ is the density of air, $\omega$ is the angular frequency and $c_{0}$ is the speed of sound in air. The boundary of the liquid film was defined as the characteristic specific acoustic impedance $\rho_{L} c_{L}$, where $\rho_{L}$ and $c_{L}$ are the liquid density and sound velocity in the liquid respectively.

The initial condition was obtained by the normal acceleration of the emitter

$$
n \cdot\left(\frac{1}{\rho_{0}}(\nabla p)\right)=a_{0}
$$

where $a_{0}$ is the normal acceleration. The parameters used for the calculation are listed in Table 2.

Table 2. Parameters used in the Comsol simulation.

\begin{tabular}{cc}
\hline Parameter & Value \\
\hline$f_{0}$ & $20.7 \mathrm{kHz}$ \\
$c_{0}$ & $343 \mathrm{~m} / \mathrm{s}$ \\
$\rho_{0}$ & $1.29 \mathrm{~kg} / \mathrm{m}^{3}$ \\
$\rho_{\mathrm{L}}$ & $994.2 \mathrm{~kg} / \mathrm{m}^{3}$ \\
$c_{\mathrm{L}}$ & $1450 \mathrm{~m} / \mathrm{s}$ \\
$a_{0}$ & $0.79 \times 10^{6} \mathrm{~m} / \mathrm{s}^{2}$ \\
\hline
\end{tabular}

\section{Calculation of acoustic radiation pressure}

The weight of the levitated sample is balanced by the acoustic radiation force which is the integral of the acoustic radiation pressure $P_{\mathrm{A}}$ over the entire surface of the sample. $P_{\mathrm{A}}$ on the sample surface was calculated by applying the obtained sound pressure according to King's theory[18]: 


$$
P_{\mathrm{A}}=\frac{1}{2 \rho_{0} c_{0}^{2}}\left\langle p^{2}\right\rangle-\frac{1}{2} \rho_{0}\left\langle v^{2}\right\rangle
$$

where $v$ is the medium particle velocity. The angular brackets in eq. (4) denote the time average over one period of acoustic oscillation. To conveniently obtain the acoustic force, a two-dimensional axisymmetric model was applied in the simulation.

In the simulation, the drop was fixed at its levitation position which can be obtained from the analysis of images recorded by the high speed camera. This is reasonable because the time-averaged acoustic radiation force can excellently balance gravity thus achieving stable levitation which has been evidenced by experiments. Even $\underline{\text { though the acoustic radiation force is time dependent, its time period is too short ( } 1 / f \text {, }}$ $\sim 0.05 \mathrm{~ms}$ ) for the levitated drop to respond, i.e. it only feels the time-averaged acoustic radiation force determined by Eq (4).

\section{Results and discussion}

\section{A. Flatting and buckling upon increasing sound intensity}

Upon increasing the sound intensity, a levitated SDS drop at its cmc can vary from an ellipsoidal shape to a thin liquid sheet (Figure 1a). To better interpret how drop flattening relates to the sound intensity, the aspect ratio $a / b$ (defined in Figure $1 b$ ) is plotted as a function of sound pressure level (SPL), which shows that $a / b$ does not increase monotonically upon increasing SPL. However, a peak appears at SPL 133.1 $\mathrm{dB}$, indicating the buckling of the flattened film at sufficiently high sound intensity.

The shape of a free drop in a gravitational field is determined by the competition between gravity and its surface tension, which is defined as the Bond number 
$B_{o}=\frac{\rho g l_{c}^{2}}{\sigma}$ (where $g$ is the gravitational acceleration and $l_{\mathrm{c}}$ is the capillary length of the liquid). Analogous to the Bond number in a gravitational field, an acoustic Bond number $B_{a}$ can be defined as[8,19]:

$$
B_{a}=2 v_{r m s}^{2} \rho_{0} R_{S} / \sigma
$$

where $v_{\text {rms }}$ is the root mean square particle velocity of the surrounding fluid and $R_{\mathrm{S}}$ is the spherical radius of the drop. The definition of $B_{\mathrm{a}}$ gives the ratio between the acoustic force and surface tension, which has an upper limit of $B_{a}{ }^{*}$ between 2.5 and 3.6[20]. This indicates that with an increase of sound intensity ( $\left.v_{r m s}\right), R_{S}$ has to reduce. Based on this concept, we thus can define an acoustic capillary length $l_{c, a}=\sigma / 2 v_{r m s}^{2} \rho_{0} R_{S}$, which decreases with increasing sound intensity. This is the main reason for the flattening of the drop. It should be noted that gravity only influences the levitation position of the drop and plays little role in determining its shape[21].

(a)

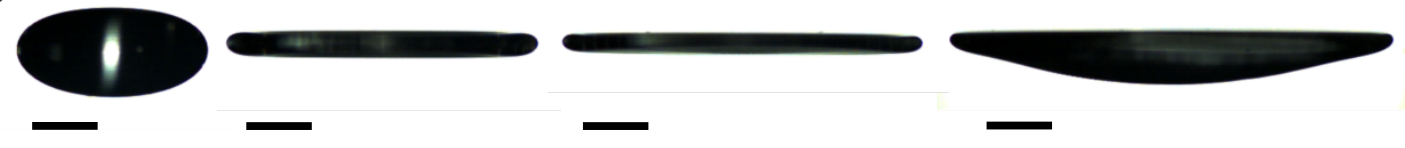

(b)
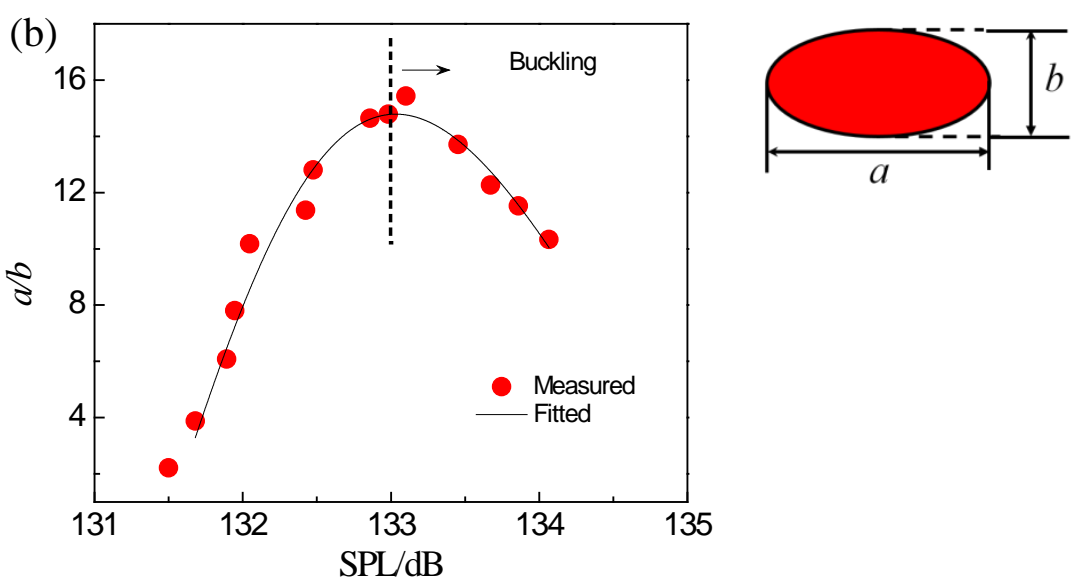

Figure 1. Shape evolution of an SDS drop (1 cmc, $10 \mu \mathrm{L})$ upon increasing sound 
intensity with decrease of emitter-reflector distance $D$. (a) Static shapes corresponding to different sound pressure levels (SPL), from left to right being $131.5 \mathrm{~dB}, 132.4 \mathrm{~dB}$, $133.1 \mathrm{~dB}$ and $134.1 \mathrm{~dB}$. Scale bars represent $1 \mathrm{~mm}$. (b) Aspect ratio $a / b$ as a function of $S P L$

It is often evidenced that internal flow appeared inside acoustically levitated drops[22, 23]. The internal flow indeed may play an important role in some dynamic behavior of the levitated drops, such as sectional oscillation [23] and drop coalescence[24]. However, it does not influence significantly the static shape of the levitated drop, where the competition between surface tension and acoustic radiation pressure dominates. One may argue that the internal flow of the drop may lead to the re-distribution of surfactant on the drop surface, and in turn affect the shape of the drop. This probably occurs for drops of low surfactant concentration. However, in the present work, we focus on surfactant of high concentrations ( $>$ cmc) which ensures sufficient fast mass transfer between the drop surface and bulk because of the presence of micelles, thus inhibiting the re-distribution.

A common phenomenon associated with flattening is buckling of the liquid film, which occurred robustly upon increasing the sound intensity. But it should be noted that the buckling direction is dependent on the levitation position, i.e. at which pressure node the liquid drop was levitated. The liquid film buckled upward when levitated at position A whereas it buckled downward at position B (Figure 2a). When the liquid film becomes sufficiently thin, it tends to follow the equipotential surface of sound field, 
and the buckling direction is determined by the curvature of the equipotential surfaces[25, 26]. In addition, the acoustic streaming may also contribute to the initial buckling. For a spherical drop, acoustic streaming has no noticeable influence on the force balance established between gravity, acoustic radiation pressure and surface tension. Whereas for the case of a thin film, the local pressure change caused by the acoustic streaming plays a role in the surface curvature. As illustrated in Figure 2b, the downward streaming may exert a push force at the center of the upper surface, leading to upward buckling. By contrast, when levitated at position $\mathrm{B}$, the upward streaming pushes the center of the lower surface which results in a reversed buckling direction (Figure 2c).
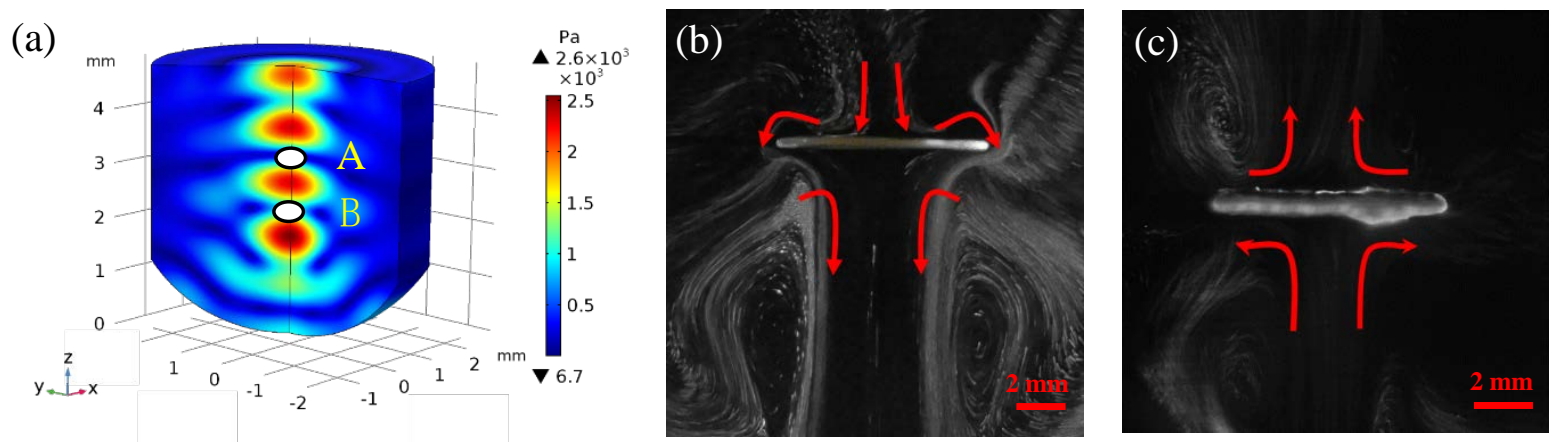

(d)

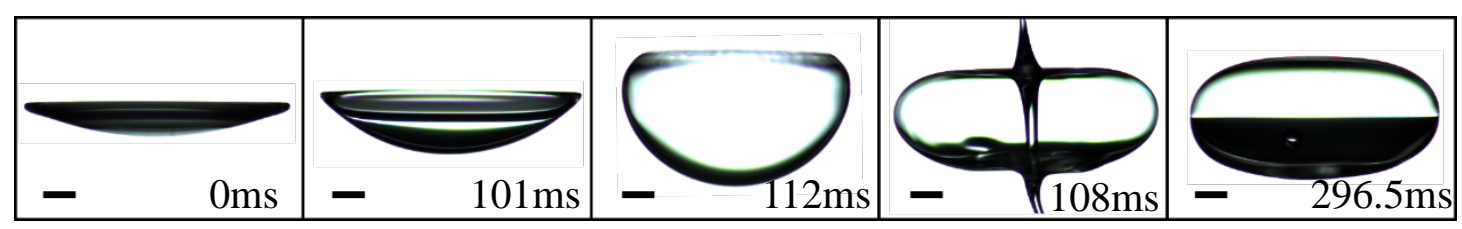

(e)

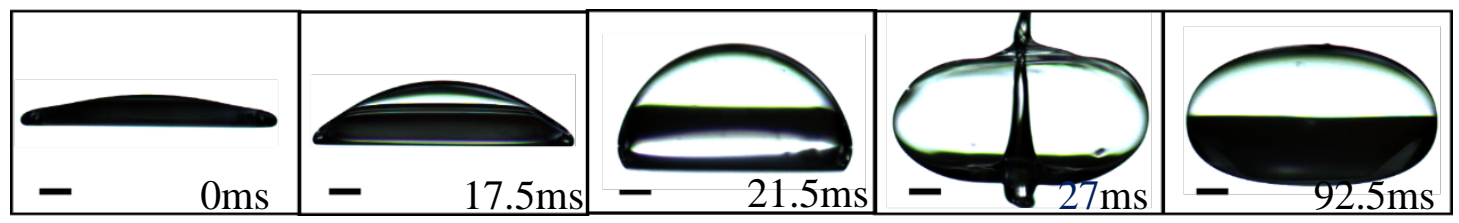

Figure 2. Buckling and bubble formation of an acoustically levitated liquid film (silicone oil $10 \mu \mathrm{L}$ ). (a) Illustration of the levitation positions in the sound field. (b), (c) 
Acoustic streaming with an extremely flattened film just before buckling levitated at positions A and B respectively. (d), (e) Snapshots showing bubble formation of drops at levitation positions A and B respectively. Scale bars represent $1 \mathrm{~mm}$.

\section{B. Bubble formation of acoustically levitated liquid film}

With further increase in sound intensity, the buckled liquid film exhibited an abrupt area expansion and rapid closure of the film rim associated with fast jetting. Eventually, a stable and closed air bubble was formed in the levitator (Figure 2d and e). The buckling direction is determined by the levitation position. However, the abrupt area expansion and rapid closure were independent of the buckling direction. The bubble formation phenomenon occurs in many different systems, ranging from a liquid crystal (5CB) to a silicone oil to aqueous surfactant solutions. Moreover, the phenomenon is not sensitive to the original volume of the drop; the volume of the final obtained bubble was almost the same despite the initial drop volume varying from 7 to $20 \mu \mathrm{L}$ (Figure 3b).

To form a stable bubble under acoustic levitation, it is of great importance that the liquid drop either possess an appropriate viscosity or is stabilized by surfactant. Pure water droplets could not transform into bubbles upon increasing the sound intensity because atomization occurs prior to the buckling transition (Figure 4a). In the presence of surfactant however, atomization can be significantly inhibited because of the Gibbs elasticity of the surfactant monolayer at the air-water interface[27].The preferred 
volume to form bubbles was found to be in the range of 7-20 $\mu \mathrm{L}$. It was found that if the volume was less than $7 \mu \mathrm{L}$, the buckled liquid film often shattered before it formed a bubble as the films became too thin (Figure 4b). For the liquid Ga-In-Sn alloy, buckling also appeared upon increasing the sound intensity. However, the rapid closure of the film rim was not observed; instead the broken of the film at its bottom (Figure 4c).

(a)

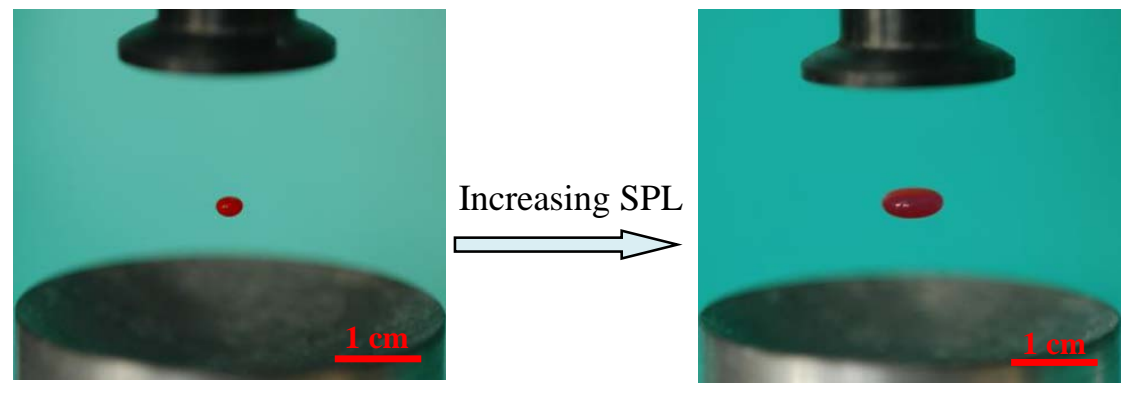

(b)

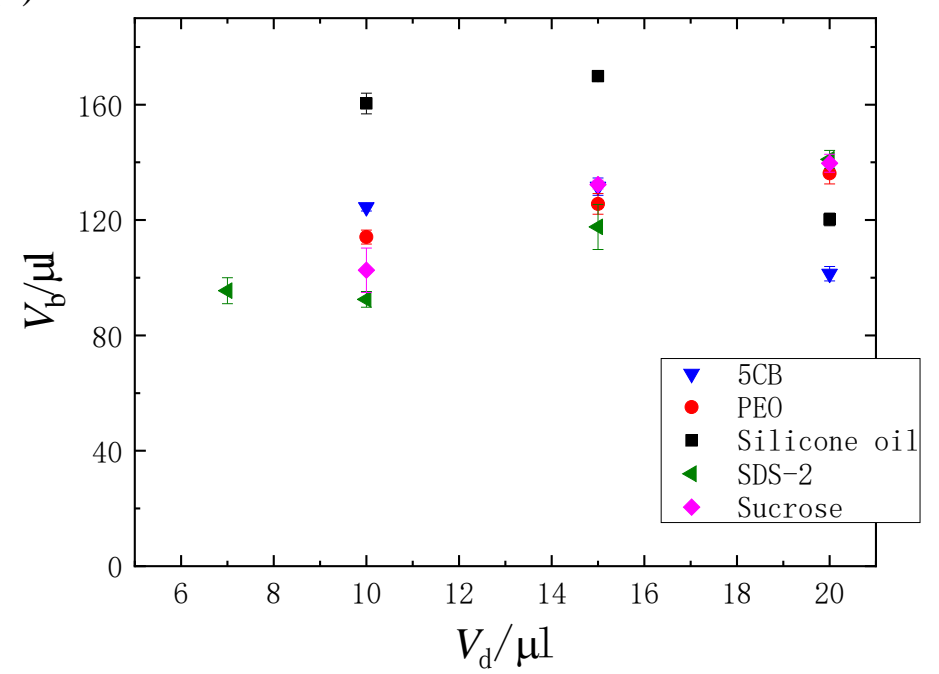

Figure 3. Final bubble volume for different volumes of liquid drops. (a) Photos showing the obtained bubble (right) is significantly larger than the initial drop (left). (b) Volume of the obtained bubble, $V_{\mathrm{b}}$ versus the initial drop volume $V_{d}$, showing $V_{\mathrm{b}}$ is virtually independent of $V_{\mathrm{d}}$. 
(a)

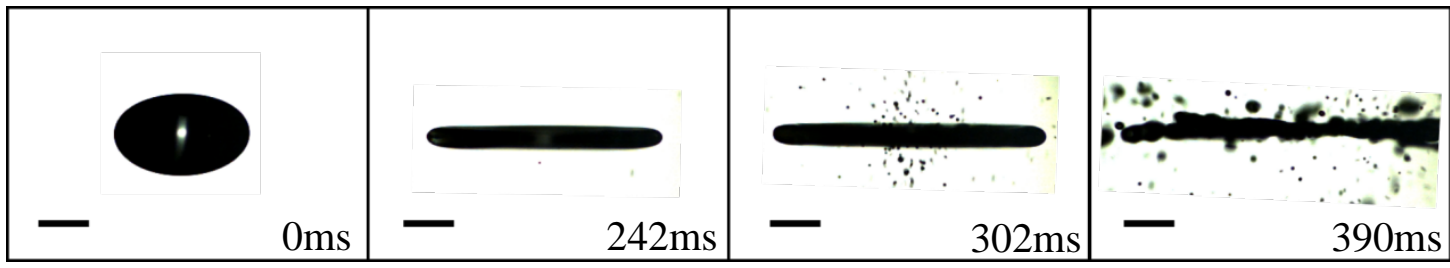

(b)

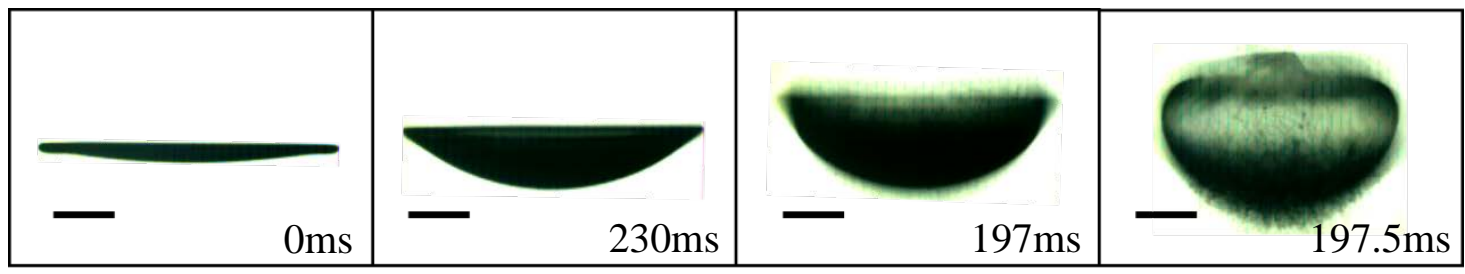

(c)

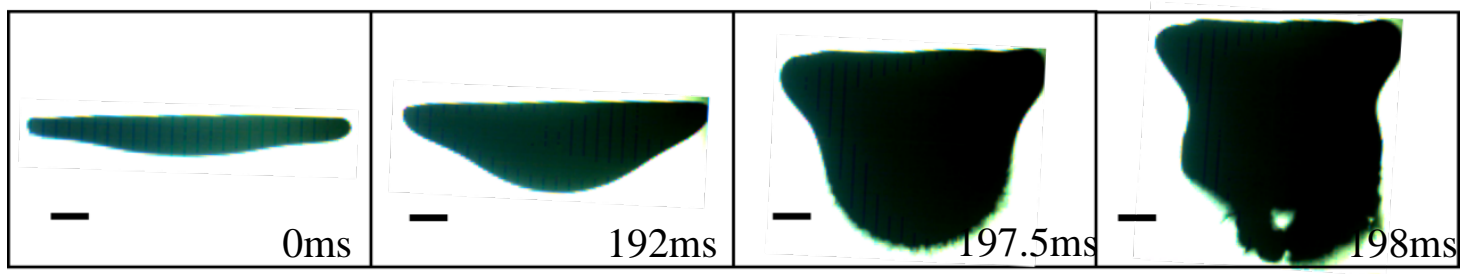

Figure 4. Experimental observations for the cases not proceeding to bubble formation. (a) Atomization prior to buckling (water, $5 \mu \mathrm{L}$ ); (b) atomization during buckling (SDS2, $5 \mu \mathrm{L}$ ); (c) film broken without closure (liquid Ga-In-Sn alloy, $10 \mu \mathrm{L}$ ). Scale bars represent $1 \mathrm{~mm}$.

The bubble formation phenomenon occurs robustly upon increasing the sound intensity. A very important feature of the phenomenon is the abrupt area expansion of the acoustically levitated liquid film once it buckled to a critical degree. In our previous work[15], we proposed an acoustic resonance mechanism to account for bubble formation, where the opening cavity encapsulated by the buckled liquid film serves as a soft sound resonator[28, 29]. The characteristic frequency of the resonator is determined by its volume, which increases with the enhancing buckling. Once the resonator reaches the critical volume, it resonates with the sound field in the levitator 
leading to the cavity inflation and bubble formation. As a result, the sound intensity in the levitator starts to decrease since the energy absorption is significantly enhanced due to resonance. This also explains the previous experimental observation that sound pressure around the levitated sample does not monotonically increase with input voltage[8].

Once the bubble was formed, it was levitated as a hollow ball with a rigid shell[30]. Its shape/aspect ratio can be tuned though adjusting the sound intensity by reducing or increasing the emitter-reflector distance [15]. However, it cannot recover to a drop anymore, i.e. the drop to bubble transition is irreversible.

\section{Sound field simulation and acoustic radiation force}

Although the acoustic resonance mechanism described the phenomenon well from the energy point of view, gaining insightful understanding based on force analysis is still desirable. Therefore, we calculated numerically the sound field and acoustic radiation pressure on the drop surface to clarify how the distribution of $P_{\mathrm{A}}$ depends on the drop shape and, in turn, influence its shape evolution. For the drop levitated at position A, the sound pressure distribution and the corresponding acoustic radiation pressure are illustrated in Figures 5 and 6, respectively. Figure 5 clearly shows the spatial distribution of sound pressure nodes and antinodes. The drop was levitated at one of the

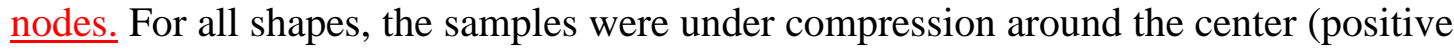
$P_{\mathrm{A}}$ ) and experienced suction around the edges (negative $P_{\mathrm{A}}$ ), as shown in Figure 6, which is consistent with previous studies[31, 32]. When the liquid drop was less 
deformed, $P_{\mathrm{A}}$ on the lower surface was larger than that on the upper surface, indicating that the drop was pushed upward by the ultrasound to balance against gravity. The dominance of $P_{\mathrm{A}}$ on the lower surface was not maintained, however, when the drop was deformed to an extremely thin film; in that case, $P_{\mathrm{A}}$ on the upper surface became dominant.

The acoustic radiation pressure difference $\Delta P_{\mathrm{A}}$ (which is greater than the Laplace pressure $4 \sigma / R=40 \mathrm{~Pa}$, for SDS-2) between the upper and lower surfaces accounted for the buckling of the film. It should be noted that at levitation position $\mathrm{B}, P_{\mathrm{A}}$ at the lower was always greater than that on the upper surface (Figure 7), consistent with the experimental results where the reversed buckling direction was consistently observed.

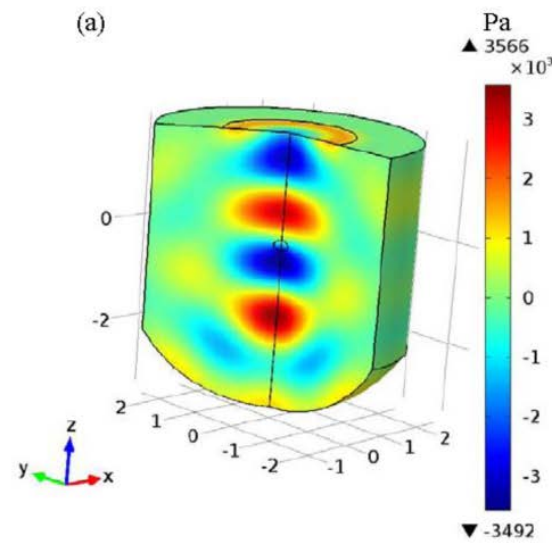

(c)

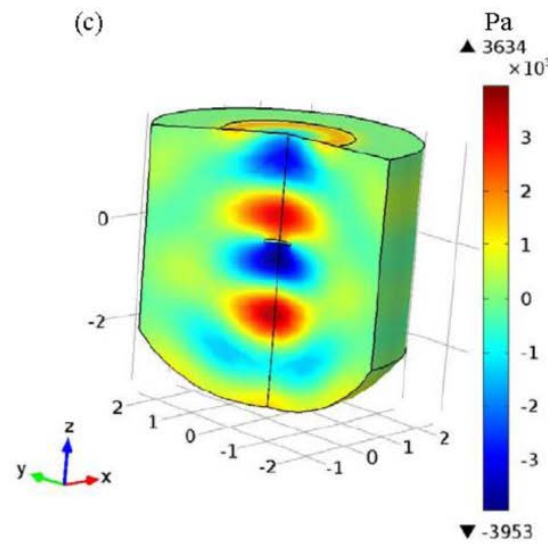

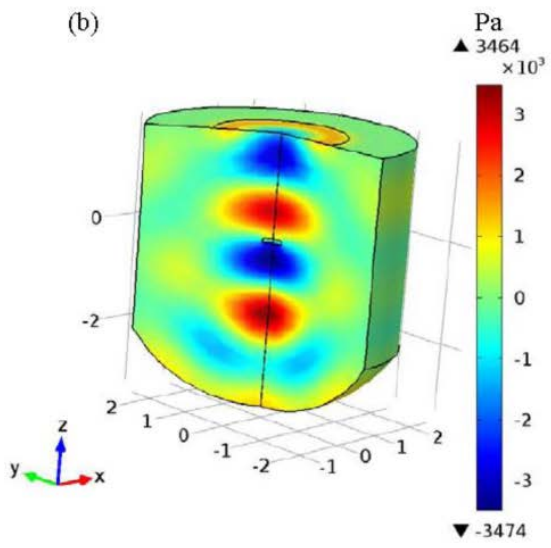

(d)

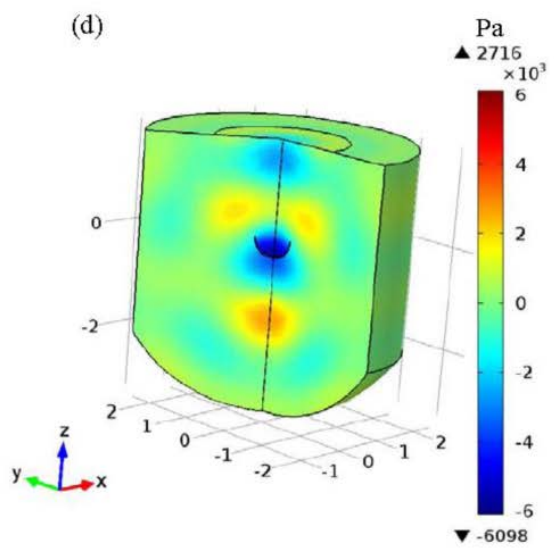

Figure 5. Sound field in the levitator with a liquid drop (SDS-2, $10 \mu \mathrm{L}$ ) levitated at 
position A (marked in Figure 2a). (a)-(d) correspond to different sample shapes: quasispherical(a), pancake-like(b), flat film(c) and buckled film(d).

(a)
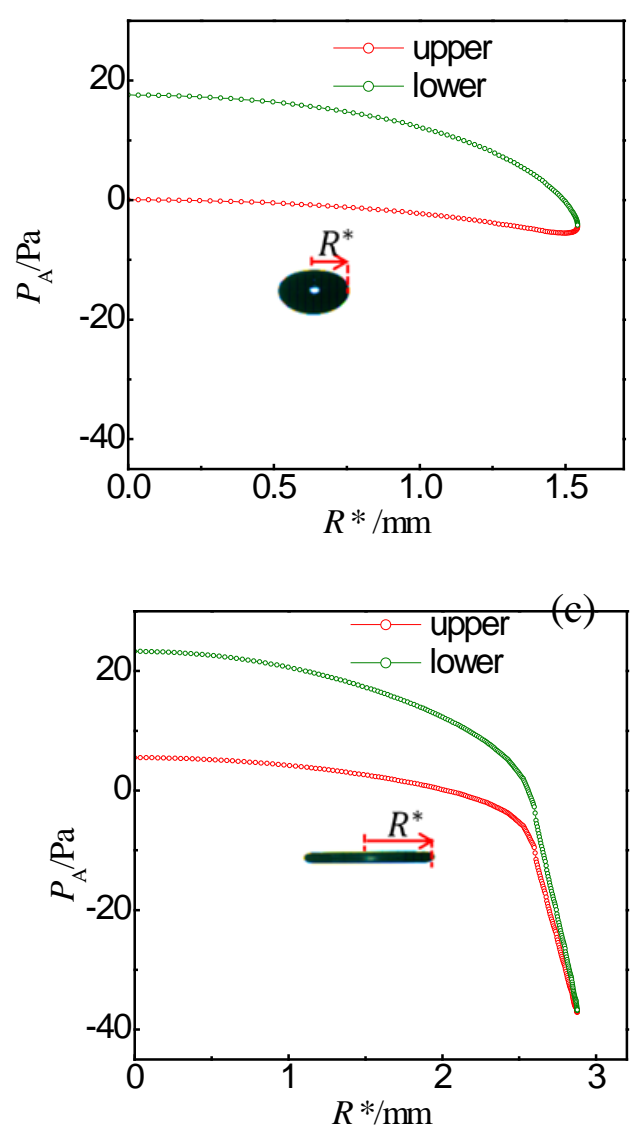

(b)

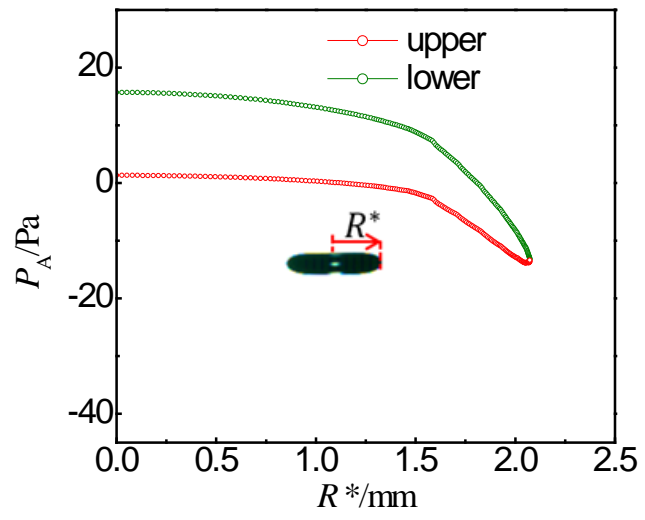

(d)

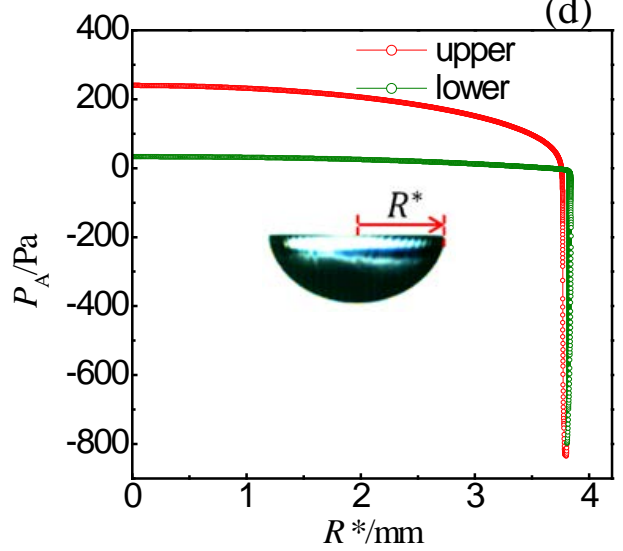

Figure 6. Acoustic radiation pressure $P_{\text {A }}$ exerted on the drop surfaces (SDS-2, $10 \mu \mathrm{L}$ ).

(a)-(d) Distribution of $P_{\mathrm{A}}$ for different shaped samples given in Figure 5. Calculations indicate that the sample was under compression at the film center and under suction at the rim. The suction effect was significantly enhanced as it was buckled.
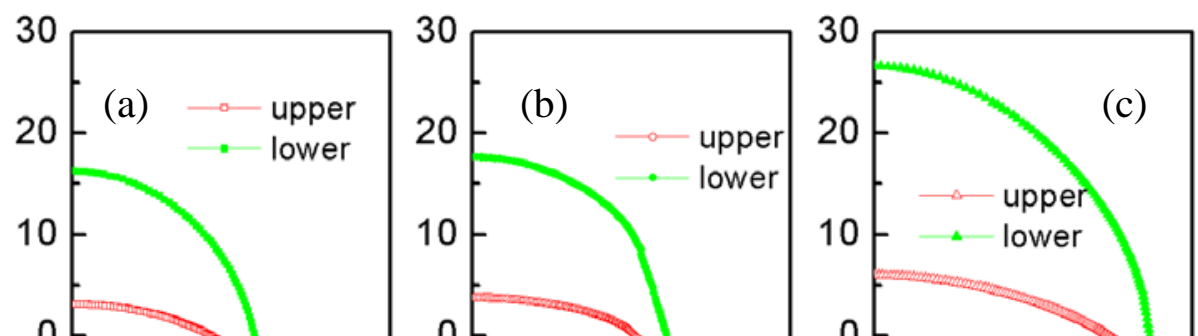
Figure 7. Distribution of $P_{\mathrm{A}}$ for different shaped liquid drop (10 $\mu \mathrm{L}$, SDS drop) levitated at position B marked in Fig.2a. (a)-(f) correspond to different sample shapes as shown in the inset graphics. $P_{\mathrm{A}}$ on the lower surface is always larger than that on the upper surface, which is consistent with the downward buckling direction observed in the experiments. The suction effect at the membrane rim is greatly enhanced once buckling occurs which is similar to results obtained at position A. 
To understand more clearly the force exerted on the acoustically levitated buckled film, the $P_{\mathrm{A}}$ distribution was schematically illustrated in Figure 8(for the case of levitated at position A), showing that the film was depressed downward on most of its surface area but dragged upward by the suction effect at its rim. The pressure distribution can also be reflected by the shape of the liquid film, which was supposed under quasi-static condition. The Laplace pressure between the inner and outer surface can be written as $\Delta P_{\mathrm{L}}=4 \sigma / R$, where $R$ is the radius of curvature. For the case of upward buckling, the downward force $F_{\mathrm{d}}$ can be estimated as:

$$
F_{d}=\Delta P_{L} \cdot \pi \cdot(R \sin \theta)^{2}=4 \pi \cdot \sigma R \sin ^{2} \theta
$$

For $\theta=45^{\circ}, R=5 \mathrm{~mm}$ and $\sigma=30 \mathrm{mN} / \mathrm{m}, F_{\mathrm{d}}$ is of magnitude $\sim 10^{-3} \mathrm{~N}$, while the gravitational force on the droplet $(10 \mu \mathrm{L})$ is $\sim 10^{-4} \mathrm{~N}$. This shows that the acoustic force causing film buckling is one order of magnitude larger than gravity and becomes the dominant force for the ultrasound to balance and to achieve stable levitation. This explains why the suction effect at the film rim was so strongly enhanced, from $-40 \mathrm{~Pa}$ to $-800 \mathrm{~Pa}$ as shown in Figure 6c-d after buckling. 


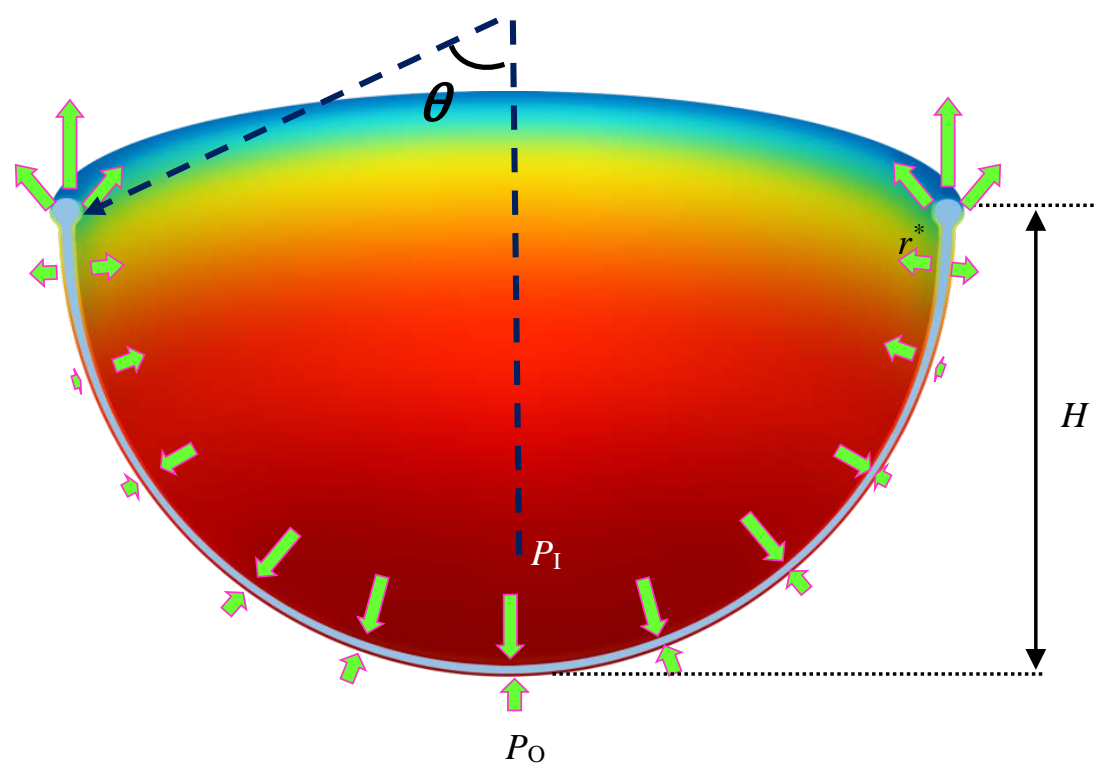

Figure 8. Color-coded illustration of the acoustic radiation pressure $P_{\mathrm{A}}$ on a buckled liquid film. Red and blue represent positive and negative values of $P_{\mathrm{A}}$, respectively. As the color of the membrane surface changed from blue to red from rim to center, $P_{\mathrm{A}}$ changed from negative to positive. Arrows indicate the directions of the acoustic radiation force, where $P_{\mathrm{I}}, P_{\mathrm{O}}$ are the acoustic radiation pressure at the inner and outer surface measured at the bottom of the buckled film, $H$ is the height of the film rim. Acoustic radiation pressure distribution shows where the film was under downward compression force at the center and upward pulling force at the rim.

\section{Scaling analysis}

To better understand the dynamics of the drop-to-bubble transition, we analyzed the time-dependent surface area of the drop/film using Pappus' theorem. We clearly observed that as the sound intensity increased, the surface area variation of an aqueous SDS drop was divisible into five different stages: slight deformation, rapid flattening, 
slow flattening, buckling and finally abrupt expansion with rim closure (Figure 9a). There was a very sharp area increase between stages 4 and 5, indicating the onset of the drop-to-bubble transition. Similar shape evolution and surface variation stages have been observed for other drops[15].

To establish a quantitative understanding of the drop-to-bubble transition (i.e. the triggering of stage 5 shown in Figure 9(a)), we performed a force analysis based on hydrostatic balance (because the process was quasi-static prior to stage 5) to estimate the critical condition under which bubble formation was initiated. For a steady, acoustically levitated liquid drop, a force balance at the drop edge can be established between the internal pressure, external suction pressure $P_{\mathrm{S}}$ and the Laplace pressure induced by the curvature of the drop rim[5]. The two former pressures tend to expand the surface area whereas the last one acts to restrain the surface expansion. For a buckled liquid film (Figure 8), the pressure balance can be written as follows

$$
P_{\mathrm{I}}-\frac{2 \sigma}{R}-\rho g H=P_{\mathrm{O}}+\frac{2 \sigma}{R}-\rho g H=P_{\mathrm{S}}+\sigma\left(\frac{1}{r^{*}}+\frac{1}{R^{*}}\right)
$$

where $H$ is the height of the film rim measured from its bottom (Figure 8 ), $R^{*}$ is the opening radius of the bowl-shaped film (Figure 6 ), $r^{*}$ is the radius of the film rim and $P_{\mathrm{I}}$ and $P_{\mathrm{O}}$ are the pressures produced by the sound field on the upper and lower surfaces, respectively, at the film bottom. Because $r^{*}$ (of the order of $10^{2} \mu \mathrm{m}$ ) is about two orders of magnitude smaller than $R, R^{*}$ and $H$, the Laplace pressure induced by film rim curvature, $\sigma / r^{*}$, plays the dominant role in restraining film expansion. Therefore, eq. (7) can be simplified as follows: 


$$
P_{\mathrm{I}}-P_{\mathrm{S}}=\Delta P: \sigma / r^{*}
$$

(a)

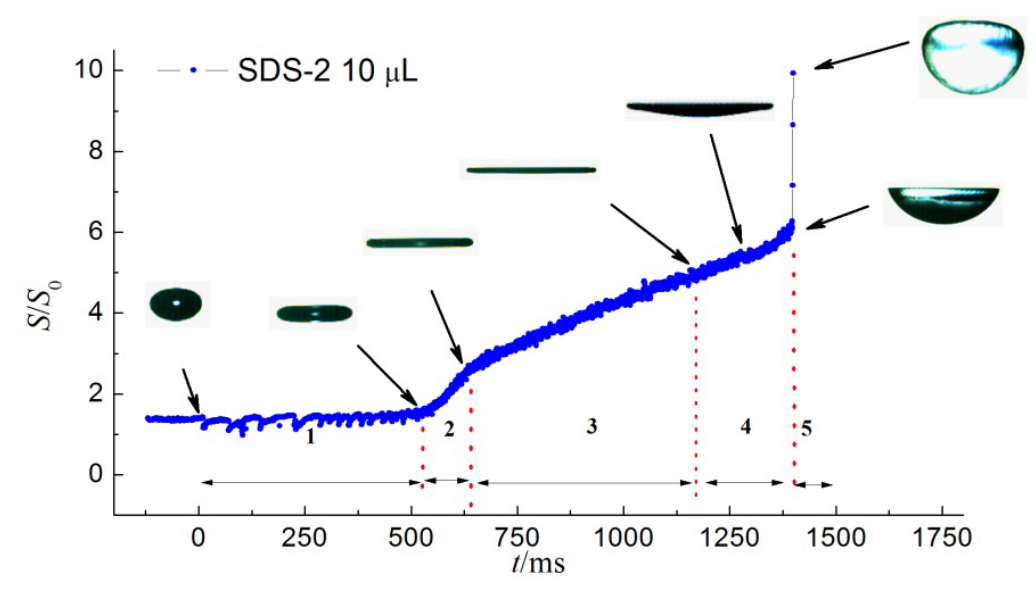

(b)

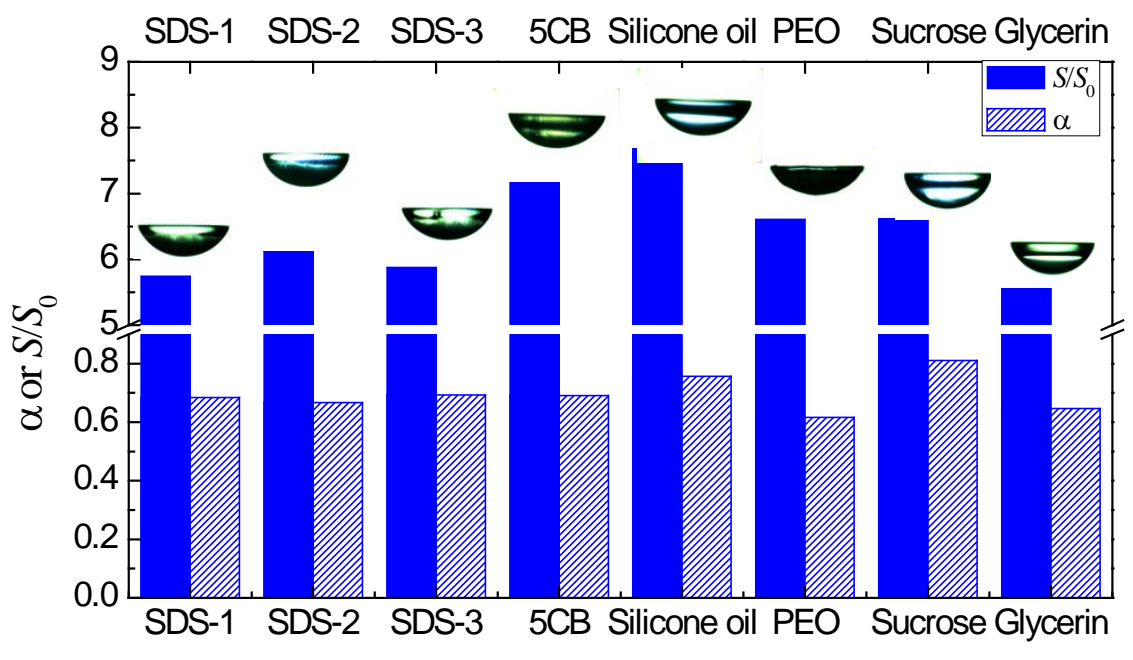

(c)

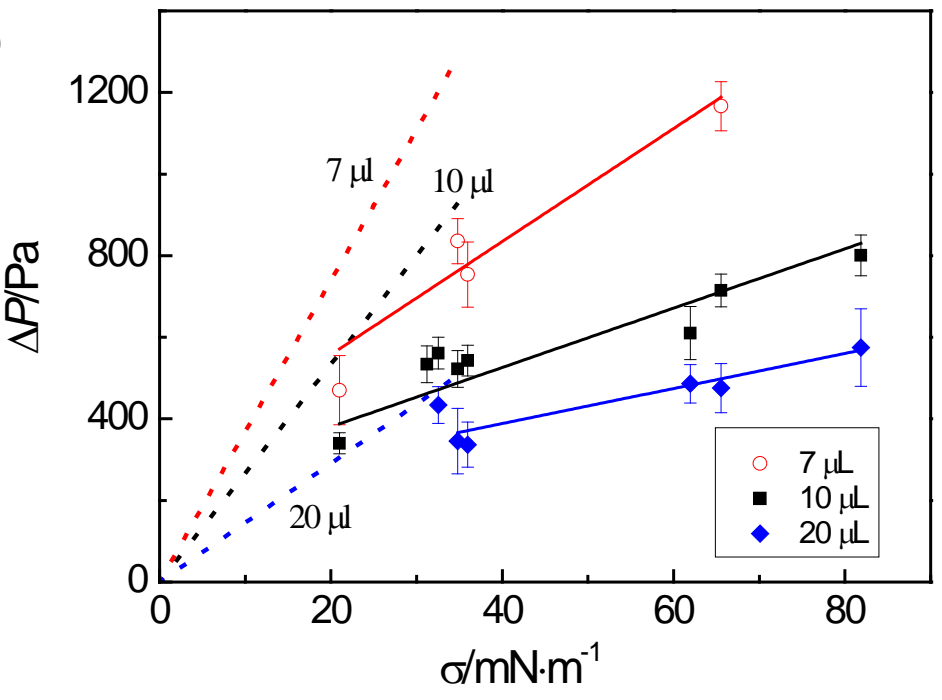


Figure 9. Bubble formation for different liquid drops. (a) Surface area variation upon increasing sound intensity of an aqueous SDS 1 drop $(10 \mu \mathrm{L})$ divided into five stages: 1-5 correspond to slight deformation, rapid flattening, slow flattening, buckling and abrupt expansion with rim closure, respectively. $t=0$ corresponds to the onset time of decreasing the emitter-reflector distance $\left(u_{\mathrm{R}}=1.00 \mathrm{~m} / \mathrm{s}\right)$. Area was scaled to the initial surface area of a spherical drop $\left(S_{0}\right)$. Inset photos show side-view snapshots corresponding to each stage. (b) Critical shape characterized by $\alpha$ or $S / S_{0}$ of the buckled film for different liquids $(10 \mu \mathrm{L})$ corresponding to the onset of abrupt area expansion for samples levitated at position A. $\alpha$ is defined as the ratio between the height $H$ of the buckled film and its radius $R^{*}$. (c) Critical driving pressure $\Delta P$ versus surface tension $\sigma$ for different liquid drops of varied volumes. The solid lines represent the linear fitting for each volume and indicate the critical driving pressure beyond which abrupt area expansion is triggered. The dashed lines indicate the slope of $2 / h$ corresponding to each volume, where $h$ is film thickness.

To verify the model in eq. (8), we numerically calculated the driving force $\Delta P$ for the onset of area expansion which corresponds to a critical geometry. The critical geometry for different liquids is similar; all show a bowl-shape (Figure 9b). However, they require different emitter-reflector distances and thus different sound intensities. The driving pressure $\Delta P$ values are plotted as a function of the surface tension $\sigma$ in Figure 9(c); they show a linear relationship for each drop volume, though the slope is much smaller than $2 / h$ confirming that the rim diameter is larger than $h$. The linear 
relationship with $\Delta P$ is larger for smaller drops, suggesting that the process is indeed controlled by surface tension and that $\Delta P \sim \sigma / r^{*}$ is the major part of the driving force for the drop-to-bubble transition. The results also indicate a non-zero intercept, representing the hydrostatic pressure required to achieve film buckling which is essential to cause the significant enhancement of suction at the film rim.

The rim dynamics during its closure was not included in our model because it plays only a minor role in triggering the drop-to-bubble transition. Rapid rim closure occurs after the transition at a velocity of $\sim 3 \mathrm{~m} / \mathrm{s}$ which is much higher than the velocity described by the Taylor relation [33] $\left.(2 \sigma / \rho h)^{1 / 2},<1 \mathrm{~m} / \mathrm{s}\right)$. This indicates that the driving force for rim dynamics is acoustic radiation pressure and not surface tension, which is time-dependent due to the coupling effect with the liquid film. The phenomenon has been observed in a wide viscosity range over two orders of magnitude ( 1-100 mPa s) and for different liquids. It seems that viscosity does not play a dominant role in the rim dynamics because the rapid closure process has a considerably large Reynolds number ( 300). However, the shear rate ${ }^{g}: R^{*} / R^{*}\left(\sim 10^{3} \mathrm{~s}^{-1}\right.$, where $R^{\mathrm{g}}$ is its closing velocity[34]) was large enough to potentially arouse a non-Newtonian effect, e.g. increasing the elongational viscosity in the presence of PEO[35], which may influence the subsequent jetting behavior.

\section{Conclusions}

In summary, we have studied the shape evolution and bubble formation of 
acoustically levitated drops based on both experimental observations and numerical simulation. Drop flattening can be caused by increasing the sound intensity and buckling occurs for extremely flattened liquid films because of following the sound filed equipotential surface and the influence of acoustic streaming. A closed bubble can be formed if the liquid drop possesses an appropriate viscosity or is stabilized by surfactant which prevents atomization during thinning. The most important finding of this study is that the buckling geometry of liquid films in the acoustic levitator caused a drastic enhanced suction effect at the film rim. A scaling analysis revealed that the driving force shows a linear relation with the surface tension. The buckling-enhanced suction effect at the film rim may also be the origin of many other processes involving ultrasound such as in ultrasound foaming[36] and emulsification[37]. Acoustic levitation of drops also provides the possibility to study the rheological properties of complex fluids by investigating the hysteresis between the drop response and the sound field change, which is highly desirable for future experimental and theoretical work.

\section{Acknowledgments}

D.Y. Zang is grateful to Prof. D. Langevin for her long term encouragement and help. We would also like to thank Prof. W.J. Xie, Prof. M.A.B. Andrade, Prof. Z.Y. Hong and Prof. L.B. Jia for their valuable input. We also thank the National Natural Science Foundation of China (Nos.U1732129 and 51672224) for financial support of this research. 


\section{References}

[1] A. A. Doinikov. Acoustic radiation forces: Classical theory and recent advances, Recent Research Developments in Acoustics 1, 39 (2003).

[2] E. H. Brandt, Levitation in Physics, Science 243, 349 (1989).

[3] Y. Bayazitoglu and G. F. Mitchell, Experiments in acoustic levitation - Surface tension measurements of deformed droplets, J. Thermophysics Heat Transfer 9, 694 (1995).

[4] J. G. Mcdaniel and R. G. Holt, Measurement of Aqueous Foam Rheology by Acoustic Levitation, Phys. Rev. E 61, R2204 (2000).

[5] P. C. Lin and L. I ?, Acoustically levitated dancing drops: Self-excited oscillation to chaotic shedding, Phys. Rev. E 93, 021101 (2016).

[6] V. Vandaele, A. Delchambre and P. Lambert, Acoustic wave levitation: Handling of components, J. Appl. Phys. 109, 124901 (2011).

[7] V. Vandaele, P. Lambert and A. Delchambre, Non-contact handling in microassembly: Acoustical levitation, Precision Engineering 29, 491 (2005).

[8] C. P. Lee, A. V. Anilkumar and T. G. Wang, Static shape and instability of an acoustically levitated liquid drop, Phys. Fluids A 3, 2497 (1991).

[9] A. B. Marco, N. P. Andrade and C. Adamowski, Experimental study of the oscillation of spheres in an acoustic levitator, J. Acoust. Soc. Am. 136, 1518 (2014).

[10] D. L. Geng, W. J. Xie, N. Yan and B. B. Wei, Vertical vibration and shape oscillation of acoustically levitated water drops, Appl. Phys. Lett. 105, 104101 (2014).

[11] D. Y. Zang, Z. C. Zhai, L. Li, K. J. Lin, X. G. Li and X. G. Geng, Vertical vibration dynamics of acoustically levitated drop containing two immiscible liquids, Appl. Phys. Lett. 109, 101602 (2016).

[12] A. V. Anilkumar, C. P. Lee, and T. G. Wang, Stability of an acoustically levitated and flattened drop-An experimental study, Phys. Fluids A 5, 2763 (1993).

[13] A. L. Yarin, D. A. Weiss, G. Brenn and D. Rensink, Acoustically levitated drops: drop oscillation and break-up driven by ultrasound modulation, Int. J. Multiphase Flow 28, 887 (2002).

[14] B. Pathak and S. Basu, Deformation pathways and breakup modes in acoustically levitated bicomponent droplets under external heating, Phys. Rev. E 93, 033103 (2016). [15] D. Y. Zang et al., Inducing drop to bubble transformation via resonance in ultrasound, Nature Commun. DOI:10.1038/s41467-018-05949-0.

[16] Z. Chen, D. Y. Zang, L. Zhao, M. F. Qu, Li X, X. G. Li, L. Lin and X. G. Geng, Liquid Marble Coalescence and Triggered Microreaction Driven by Acoustic Levitation, Langmuir 33, 6232 (2017).

[17] V. Kocourek, Ch. Karcher, M. Conrath and D. Schulze, Stability of liquid metal drops affected by a high-frequency magnetic field, Phys. Rev. E 74, 026303 (2006).

[18] L. V. King, On the acoustic radiation pressure on spheres, Proc. R. Soc. London A 147, 212 (1934).

[19] D. Foresti, M. Nabavi, M. Klingauf, A. Ferrari and D. Poulikakos, Acoustophoretic contactless transport and handling of matter, PNAS 110, 12549 (2013). 
[20] C. P. Lee, A. V. Anilkumar and T. G. Wang, Static shape of an acoustically levitated drop with wave-drop interaction, Phys. Fluids 6, 3554 (1994).

[21] W. J. Xie and B. B. Wei, Dynamics of acoustically levitated disk samples, Phys. Rev. E 70, 046611 (2004).

[22] Y. Yamamoto, Y. Abe, A. Fujiwara, K. Hasegawa and K. Aoki, Internal Flow of Acoustically Levitated Droplet, Microgravity Sci. Technol. 20, 277 (2008).

[23] C. L. Shen, W. J. Xie, Z. L. Yan and B. Wei, Internal flow of acoustically levitated drops undergoing sectorial oscillations, Phys. Lett. A 374, 4045 (2010).

[24] G. P. Neitzel and P. Dell'Aversana, Noncoalescence and nonwetting behavior of liquids, Annu. Rev. Fluid Mech. 34, 267 (2002).

[25] M. Evander and J. Nilsson, Acoustofluidics 20: applications in acoustic trapping, Lab on a chip 12, 4667 (2012).

[26] F. J. Trujillo, P. Juliano, G. Barbosa-Canovas and K. Knoerzer, Separation of suspensions and emulsions via ultrasonic standing waves - a review, Ultrason. Sonochem. 21, 2151 (2014).

[27] E. H. Lucassen-Reynders, A. Cagna and J. Lucassen, surface dilational modulus and diffusional relaxation in nonionic surfactant monolayers, Colloids Surf. A 186, 63 (2001).

[28] N. Fang, D. J. Xi, J. Y. Xu, M. Ambati, W. Srituravanich, C. Sun and X. Zhang, Ultrasonic metamaterials with negative modulus, Nature Mater. 5, 452 (2006).

[29] C. L. Ding, H. J. Chen, S. L. Zhai, S. Liu and X. P. Zhao, The anomalous manipulation of acoustic waves based on planar metasurface with split hollow sphere, J. Phys. D: Appl. Phys. 48, 045303 (2015).

[30] D. Y. Zang, K. J. Lin, L. Li, Z. Chen, X. G. Li and X. G. Geng, Acoustic levitation of soap bubbles in air: beyond the half-wavelength limit of sound, Appl. Phys. Lett. 110, 121602 (2017).

[31] W. J. Xie, C. D. Cao, Y. J. Lü, Z. Y. Hong and B. B. Wei, Acoustic method for levitation of small living animals, Appl. Phys. Lett. 89, 214102 (2006).

[32] D. Y. Zang, J. Li, Z. Chen, Z. C. Zhai, X. G. Geng and B. P. Binks, Switchable Opening and Closing of a Liquid Marble via Ultrasonic Levitation, Langmuir 31, 11502 (2015).

[33] G. I. Taylor, The Dynamics of Thin Sheets of Fluid. II. Waves on Fluid Sheets, Proc. R. Soc. London A 253, 296 (1959).

[34] G. Debregeas, P.-G. de Gennes and F. Brochard-Wyart, Science, The Life and Death of "Bare” Viscous Bubbles, Science 279, 1704 (1998).

[35] C. Wagner, Y. Amarouchene, D. Bonn and J. Eggers, Droplet detachment and satellite bead formation in viscoelastic fluids, Phys. Rev. Lett. 95, 164504 (2005).

[36] A. Gandhi, N. Asija, K. K. Gaur, S. J. A. Rizvi, V. Tiwari and N. Bhatnagar, Ultrasound assisted cyclic solid-state foaming for fabricating ultra-low density porous acrylonitrile-butadiene-styrene foams, Mater. Lett. 94, 76 (2013).

[37] S. G. Gaikwad and A. B. Pandit, Ultrasound emulsification: effect of ultrasonic and physicochemical properties on dispersed phase volume and droplet size, Ultrason. Sonochem. 15, 554 (2008). 